\title{
Sources of Trophic Action on Performance and Intestinal Morphometry of Broiler Chickens Vaccinated against Coccidiosis
}

http://dx.doi.org/10.1590/1516-635x1604389-396

\section{-Author(s)}

Sakamoto Ml

Faria DE'

Nakagi VS'

Murakami AE"

Faculdade de Zootecnia e Engenharia de Alimentos da Universidade de São Paulo (FZEA/USP). Departamento de Zootecnia. Av. Duque de Caxias Norte, 225 CEP.13635-900 - Pirassununga, SP - Brasil;

" Universidade Estadual de Maringá (UEM). Departamento de Zootecnia. Av. Colombo 5790 - CEP.87020-900 - Maringá, Pr Brasil.

\section{Mail Address}

Corresponding author e-mail address E-mail: mizumiss@yahoo.com.br

\section{nKeywords}

Eimeria, glutamine, intestinal mucosa, nucleotides.

\section{ABSTRACT}

The aim of this experiment was to evaluate the effect of sources of trophic action (glutamine alone, glutamine associated with glutamic acid and yeast) associated with vaccination against coccidiosis on the performance and morphometry of the small intestine of broilers. In the trial, 1,200 broiler chicks were distributed according to a completely randomized design with a $3 \times 2+2$ (trophic action $\times$ vaccination or not + control - free trophic factor) factorial arrangement, with five replicates of 30 birds each. Vaccination negatively affected performance parameters and the morphometry of the intestinal mucosa, but at the end of the experimental period, among the broilers vaccinated against coccidiosis, the group fed glutamine presented better recovery from epithelial losses of the intestinal mucosa compared with the control groups. Therefore, the dietary supplementation with the evaluated sources of trophic action could be a strategy to enhance the development of broilers submitted to vaccine stress, also considering the economic viability of the productive segment.

\section{INTRODUCTION}

Feed intake is considered a limiting factor for broiler growth, and with this respect, the development of the gastrointestinal tract (GIT) has an important role. Although the GIT is anatomically developed during the embryonic stage, considerable alterations occur after hatching, as the proliferation rate of the enterocytes increases (Macari et al., 2002).

The intestine is an important organ, responsible for the digestion, absorption and metabolism of the nutrients in the diet. It contributes with 9 to $12 \%$ of the protein synthesis of the organism and is one of the main sites where unknown antigens, including food proteins, natural toxins and invading pathogens invade the body (Li et al., 2007).

Considering the demand of the consumer market, the need for research on alternative products has arisen, such as amino acids and nucleotides, which could substitute the antibiotics preventing productivity losses and health problems in animals (Rossi et al., 2007).

Amino acids are essential substrates not only for the synthesis of proteins and other nitrogenous compounds, but also are key regulators of other metabolic fluxes (Jobgen et al., 2006). Recent studies with animals and humans indicate that some amino acids play a key role in the maintenance of intestinal health (Amin et al., 2002; Wang et al., 2008). However, little is known about the role of amino acids in the maintenance of gut barrier integrity, preventing possible enteric diseases (Wang et al., 2009).

The amino acid glutamine participates in many metabolic processes, such as protein synthesis, gluconeogenesis, nucleic acid biosynthesis, immune response, and provides support for rapidly proliferating cells, 
including enterocytes and lymphatic cells, apart from its antioxidant function (Domeneghini et al., 2006; Wu et al., 2007; Wang et al., 2008).

Nucleotides take part in cell division, cell growth and modulation of the immune system. In addition, dietary nucleotides can help in maintaining intestinal health, reducing the incidence of enteric diseases (Mateo \& Stein, 2004).

Coccidiosis is an enteric disease that can lead to considerable losses in poultry production. Annual world economic losses have been estimated as 1.5 billion dollars (Revolledo \& Ferreira, 2005), and in Brazil the annual losses are over 30 million dollars (Kawazoe, 2000). When coccidia infect a broiler flock, it is difficult to control the dissemination the disease, due to the confinement of birds and the high stocking density in the broiler house, favoring the widespread contamination of the environment with oocysts.

Therefore, the aim of the present experiment was to evaluate the effects of the dietary inclusion of different sources of trophic action, such as glutamine and yeast, on the performance and intestinal morphometry of broilers vaccinated against coccidiosis.

\section{MATERIAL AND METHODS}

The experiment was carried out in Pirassununga, state of São Paulo, Brazil, and was approved by the Animal Ethics Committee under protocol number 04/01/2007. In the experiment, 1,200 one-day-old male Cobb-Vantress ${ }^{\circledR}$ broilers, with average initial weight of 43.30 grams, were housed in a conventional broiler house.

A completely randomized experimental design in a $3 \times 2$ factorial arrangement (dietary sources of trophic action $x$ vaccination against coccidiosis) +2 control treatments was applied. Treatments consisted of: 1) glutamine (L-GIn) feed supplementation and no vaccination; 2) L-GIn and vaccination; 3) glutamine + glutamic acid ( $\mathrm{Gln}+\mathrm{Glu}$ ) feed supplementation and no vaccination; 4) Gln + Glu and vaccination; 5) yeast feed supplementation and no vaccination; 6) yeast feed supplementation and vaccination; 7) positive control (feed with no trophic factors and no vaccination); and 8) negative control (feed with no trophic factors and vaccination), giving a total of eight treatments with five replicates of 30 birds each. Trophic factors were supplemented in the feed during the first 21 days of age. The birds were vaccinated against coccidiosis (BioCoccivet- $R^{\circledR}$ ) at $5 \mathrm{~d}$ of age, via eye drop, according to the manufacturer's recommendation $(0.03 \mathrm{~mL} / \mathrm{bird})$.
The diets were based on corn and soybean meal, as shown in Table 1, and formulated to supply broilers nutritional requirements according to Rostagno et al. (2005). In the grower and finisher phases, birds were fed a same basal diet. The levels of the trophic sources used were determined in earlier experiments of these authors (Sakamoto, 2009). The glutamine sources were provided by Ajinomoto Interamericana and the yeast contained $2 \%$ nucleotides $+1 \%$ RNA $+97 \%$ Saccharomyces cerevisiae. The vitamin-mineral premix used contained no anticoccidial agent.

Average temperatures and relative humidity (maximum and minimum values) inside the broiler house were measured daily with the aid of a manual thermo-hygrometer. Average maximum and minimum temperature and relative humidity of $28.79 \pm 1.75$ and $21.26 \pm 1.26{ }^{\circ} \mathrm{C} ; 70.29 \pm 8.53$ and $45.63 \pm 7.75 \%$, respectively, were recorded during the experimental period.

Birds were vaccinated against Marek's disease at the hatchery, and against Newcastle disease and infectious bursal disease $\left(\right.$ Biovet $\left.^{\circledR}\right)$, via eye drop, at 10 days of age.

Birds and feeds were weighed in all rearing phases to determine their performance parameters (body weight - BW, body weight gain - BWG, feed intake - FI, feed:gain ratio - FG, and production efficiency index - PEI). Mortality and feed residues were also recorded daily to determine actual feed intake.

On days $7,14,21$, and 41 , two birds per replicate (selected at random) were electrically stunned and then killed by exsanguination, and eviscerated for the morphometric analyses of the intestinal mucosa. Fragments of approximately $5 \mathrm{~cm}$ in length of each of the three regions of the small intestine (duodenum: from the pylorus to the distal portion of the duodenal loop; jejunum: from the distal portion of the duodenal loop to Meckel's diverticulum; and ileum: anterior portion of the cecum), were collected for the analyses of villus height, crypt depth, and villus:crypt ratio.

Segments were longitudinally opened at the mesenteric border, washed with saline solution to remove all intestinal contents and fixed in Bouin's solution for 24 hours, and processed until paraffin embedding according to Beçak \& Paulete (1976). Each fragment was submitted to semi-seriate cuts (5 $\mu \mathrm{m}$ thick) and stained using the hematoxylin-eosin method. In the morphometric study, images were obtained using a light microscope and analyzed using a digital image processing software (AxioVision - version 4.6 - Zeiss ${ }^{\circledR}$. The height of 30 villus and the depth of 
Table 1 - Ingredients and calculated nutritional composition of the broiler diets supplemented with different trophic sources in the pre-starter phase ( 1 to $7 \mathrm{~d}$ of age) and starter phase (8 to $21 \mathrm{~d}$ of age).

\begin{tabular}{|c|c|c|c|c|c|c|c|c|}
\hline \multirow[b]{2}{*}{ Ingredients } & \multicolumn{4}{|c|}{ Pre-starter } & \multicolumn{4}{|c|}{ Starter } \\
\hline & L-Gln & $\mathrm{Gln}+\mathrm{Glu}$ & Yeast & Control & $L-G \ln$ & $\mathrm{Gln}+\mathrm{Glu}$ & Yeast & Control \\
\hline Corn & 58.549 & 55.027 & 60.896 & 62.014 & 59.380 & 58.337 & 61.264 & 63.994 \\
\hline $45 \%$ soybean meal & 33.395 & 34.289 & 32.798 & 32.500 & 31.555 & 31.821 & 31.075 & 30.380 \\
\hline L-Glutamine $^{1}$ & 1.500 & 0.000 & 0.000 & 0.000 & 1.500 & 0.000 & 0.000 & 0.000 \\
\hline $\mathrm{G} \ln +\mathrm{Glu}^{2}$ & 0.000 & 3.000 & 0.000 & 0.000 & 0.000 & 3.000 & 0.000 & 0.000 \\
\hline Yeast $^{3}$ & 0.000 & 0.000 & 0.500 & 0.000 & 0.000 & 0.000 & 0.500 & 0.000 \\
\hline Dicalcium phosphate & 1.998 & 2.005 & 1.994 & 1.992 & 1.906 & 1.907 & 1.903 & 1.897 \\
\hline Limestone & 0.937 & 0.929 & 0.943 & 0.946 & 0.804 & 0.759 & 0.807 & 0.815 \\
\hline Soybean oil & 1.737 & 2.879 & 0.976 & 0.596 & 3.591 & 2.915 & 3.104 & 1.500 \\
\hline Salt & 0.281 & 0.284 & 0.279 & 0.278 & 0.297 & 0.298 & 0.262 & 0.293 \\
\hline Sodium Bicarbonate & 0.363 & 0.360 & 0.365 & 0.366 & 0.210 & 0.210 & 0.320 & 0.300 \\
\hline 99\% DL-Met & 0.367 & 0.372 & 0.364 & 0.362 & 0.191 & 0.192 & 0.190 & 0.177 \\
\hline $79 \%$ L-Lys $\mathrm{HCl}$ & 0.525 & 0.509 & 0.536 & 0.542 & 0.325 & 0.320 & 0.333 & 0.346 \\
\hline 98.5\% L-Treo & 0.208 & 0.206 & 0.209 & 0.209 & 0.101 & 0.101 & 0.102 & 0.103 \\
\hline Choline Chloride & 0.030 & 0.030 & 0.030 & 0.030 & 0.030 & 0.030 & 0.030 & 0.030 \\
\hline Mineral premix ${ }^{4}$ & 0.050 & 0.050 & 0.050 & 0.050 & 0.050 & 0.050 & 0.050 & 0.050 \\
\hline Vitamin premix ${ }^{5}$ & 0.050 & 0.050 & 0.050 & 0.050 & 0.050 & 0.050 & 0.050 & 0.050 \\
\hline Anticoccidial $^{6}$ & 0.000 & 0.000 & 0.000 & 0.055 & 0.000 & 0.000 & 0.000 & 0.055 \\
\hline Antioxidant $^{7}$ & 0.010 & 0.010 & 0.010 & 0.010 & 0.010 & 0.010 & 0.010 & 0.010 \\
\hline Total & 100.00 & 100.00 & 100.00 & 100.00 & 100.00 & 100.00 & 100.00 & 100.00 \\
\hline \multicolumn{9}{|l|}{ Calculated levels } \\
\hline $\mathrm{ME}(\mathrm{kcal} / \mathrm{kg})$ & 2,960 & 2,960 & 2,960 & 2,960 & 3,000 & 3,000 & 3,000 & 3,000 \\
\hline Crude Protein (\%) & 22.10 & 22.10 & 22.10 & 22.10 & 21.00 & 21.00 & 21.00 & 21.00 \\
\hline Calcium (\%) & 0.98 & 0.98 & 0.98 & 0.98 & 0.90 & 0.90 & 0.90 & 0.90 \\
\hline Available Phosphorus (\%) & 0.48 & 0.48 & 0.48 & 0.48 & 0.46 & 0.46 & 0.46 & 0.46 \\
\hline Dig. Met+cys *(\%) & 0.95 & 0.95 & 0.96 & 0.96 & 0.76 & 0.76 & 0.76 & 0.75 \\
\hline Digestible Met (\%) & 0.66 & 0.66 & 0.66 & 0.66 & 0.48 & 0.48 & 0.48 & 0.47 \\
\hline Digestible Lys (\%) & 1.35 & 1.35 & 1.35 & 1.36 & 1.16 & 1.16 & 1.16 & 1.16 \\
\hline Digestible Arg (\%) & 1.25 & 1.26 & 1.25 & 1.24 & 1.20 & 1.21 & 1.20 & 1.19 \\
\hline Digestible Thr (\%) & 0.87 & 0.87 & 0.87 & 0.87 & 0.75 & 0.75 & 0.75 & 0.74 \\
\hline $\mathrm{Na}(\%)$ & 0.25 & 0.25 & 0.25 & 0.25 & 0.21 & 0.21 & 0.23 & 0.23 \\
\hline $\mathrm{Cl}(\%)$ & 0.20 & 0.20 & 0.20 & 0.20 & 0.21 & 0.21 & 0.19 & 0.21 \\
\hline K (\%) & 0.79 & 0.80 & 0.78 & 0.78 & 0.76 & 0.76 & 0.75 & 0.75 \\
\hline DEB $(\mathrm{mEq} / \mathrm{kg})^{8}$ & 254.37 & 255.88 & 253.37 & 252.86 & 227.88 & 228.33 & 239.66 & 236.09 \\
\hline
\end{tabular}

1- L-Glutamine: Analytical results - Transmittance, 98\%; Cl, 0.020\%; $\mathrm{NH}_{4}, 0.10 \% ; \mathrm{SO}_{4}, 0.020 \%$; Fe 10ppm; $\mathrm{PB}, 10$ ppm; $\mathrm{As}_{2} \mathrm{O}_{3}, 1$ ppm; loss on drying, 0,03\%; assay, 99,7\%; pH,

5,5 (Ajinomoto Interamericana Ind. e Com. Ltda., São Paulo, Brazil).

2- Gln+Glu (Aminogut $\left.{ }^{\circledR}\right)$ : L- glutamic acid (>10\%), L-glutamine (>10\%). Ajinomoto Interamericana Ind. e Com. Ltda.

3- NucleotAid ${ }^{\circledR}$ : total nucleotides (3\%); inactivated yeast (97\%). Formil Vet Ind. e Com. Ltda, São Paulo, Brazil.

4- Mineral supplement (supply per kg product): Mn, 150.000 mg; Zn, 140.000 mg; Fe, 100.000 mg; Cu, 16.000 mg; I, 1.500 mg.

5-Vitamin supplement (supply per kg product): Se, $600 \mathrm{mg}$; vitamin $\mathrm{A}$, $20.000 .000 \mathrm{IU}$; vitamin $\mathrm{D}_{3}, 5.000 .000 \mathrm{IU}$; vitamin $\mathrm{E}, 50.000 \mathrm{mg}$; vitamin $\mathrm{K}_{3}$, $4.000 \mathrm{mg}$; vitamin $\mathrm{B}_{1}, 5.000 \mathrm{mg}$; vitamin $B_{2^{\prime}} 13.000 \mathrm{mg}$; vitamin $B_{6^{\prime}} 7.000 \mathrm{mg}$; vitamin $B_{12^{\prime}} 36.000 \mathrm{mcg}$; folic acid, $2.400 \mathrm{mg}$; pantothenic acid, $30.000 \mathrm{mg}$; niacin, $84.000 \mathrm{mg}$; biotin, $160.000 \mathrm{mcg}$; ethoxyquin, $166 \mathrm{mg} ;$ assay Q.S.P.

6- Anticoccidial: pre-starter and starter phases = Coban 200 (Sodium Monensin , 20\%): 550 g/ton (110 ppm) - Elanco Saúde Animal Ltda., São Paulo, Brazil.

7- Antioxidant: BHT (Butylated -hydroxy-toluene).

8- Dietary Electrolyte Balance (Mongin, 1981) $=\left[\left(\% \mathrm{Na}^{*} 10,000 / 22.990\right)+\left(\% \mathrm{~K}^{*} 10,000 / 39.102\right)\right]-\left(\% \mathrm{Cl}{ }^{*} 10,000 / 35.453\right)$ 


\section{Sources of Trophic Action on Performance and Intestinal Morphometry of Broiler Chickens Vaccinated against Coccidiosis}

30 crypts in each segment were measured per bird. The data were submitted to analysis of variance using the General Linear Model (GLM) procedure of SAS statistical package (SAS Institute, 2002). Means were compared by the test of Tukey at $5 \%$ probability level.

\section{RESULTS}

Trophic sources influenced ( $p=0.025)$ broiler feed intake during the pre-starter phase (from 1 to $7 \mathrm{~d}$ of age), the effect being greater for L-GIn than for
Gln+Glu (160.21 g vs. 149.78 g), which, for its part, did not differ from the other treatments. There was an interaction $(p=0.017)$ of the factors analyzed for feed:gain, and the best results for the factor trophic sources were obtained with $\mathrm{G} \mid \mathrm{n}+\mathrm{Glu}$, independently of vaccination, but were not different from those obtained with the positive (non-vaccinated) control treatment, as shown in Table 2.

In the starter phase (from 1 to $21 \mathrm{~d}$ of age), all of the evaluated parameters were affected by the

Table 2 - Effects of the interactions between factors on performance parameters of broilers fed diets supplemented with different trophic sources and vaccinated (VAC) or not (NVAC) against coccidiosis.

\section{1 to $7 \mathrm{~d}$ of age}

Feed:Gain Ratio - FCR $\left(\mathrm{g} \mathrm{g}^{-1}\right)$

\begin{tabular}{|c|c|c|c|c|c|}
\hline NVAC & $1.061 \mathrm{Aa}$ & $0.982 \mathrm{Ab}$ & $1.019 \mathrm{Aab}$ & $0.990 \mathrm{Ab}$ & $1.013 \pm 0.014$ \\
\hline VAC & 1.007 Aab & $0.994 \mathrm{Ab}$ & $1.024 \mathrm{Aab}$ & $1.030 \mathrm{Aa}$ & $1.014 \pm 0.016$ \\
\hline Mean $\pm S E^{*}$ & $1.034 \pm 0.015$ & $0.988 \pm 0.012$ & $1.021 \pm 0.016$ & $1.010 \pm 0.014$ & \\
\hline \multicolumn{6}{|c|}{1 to $21 \mathrm{~d}$ of age } \\
\hline \multicolumn{6}{|c|}{ Body Weight - BW (g) } \\
\hline NVAC & $1,060.61 \mathrm{Aa}$ & $1,028.46 \mathrm{Aa}$ & $1,026.76 \mathrm{Aa}$ & $992.20 \mathrm{Ab}$ & $1,027.00 \pm 9.50$ \\
\hline VAC & $990.55 \mathrm{Ba}$ & $976.23 \mathrm{Bb}$ & $962.05 \mathrm{Bb}$ & $923.52 \mathrm{Bb}$ & $963.09 \pm 9.10$ \\
\hline Mean $\pm \mathrm{SE}^{*}$ & $1,025.58 \pm 9.545$ & $1,002.35 \pm 9.705$ & $994.40 \pm 9.387$ & $957.86 \pm 9.345$ & \\
\hline \multicolumn{6}{|c|}{ Body Weight Gain - WG (g) } \\
\hline NVAC & $1,017.14 \mathrm{Aa}$ & $985.40 \mathrm{Aab}$ & $983.02 \mathrm{Aab}$ & $949.53 \mathrm{Ab}$ & $983.77 \pm 9.45$ \\
\hline VAC & $947.23 \mathrm{Ba}$ & $932.76 \mathrm{Bab}$ & $918.58 \mathrm{Bb}$ & $880.32 \mathrm{Bb}$ & $919.73 \pm 9.42$ \\
\hline Mean $\pm \mathrm{SE}^{*}$ & $982.19 \pm 9.457$ & $959.08 \pm 9.436$ & $950.80 \pm 9.434$ & $914.92 \pm 9.427$ & \\
\hline \multicolumn{6}{|c|}{ Feed Intake - FI ( g bird $\left.^{-1}\right)$} \\
\hline NVAC & $1,327.99 \mathrm{Aa}$ & $1,316.77 \mathrm{Ab}$ & $1,351.33 \mathrm{Aa}$ & 1,324.82 Aab & $1,330.23 \pm 16.53$ \\
\hline VAC & $1,316.26 \mathrm{Ba}$ & $1,297.98 \mathrm{Bb}$ & $1,302.30 \mathrm{Ba}$ & 1,299.50 Bab & $1,304.01 \pm 16.23$ \\
\hline Mean $\pm \mathrm{SE}^{*}$ & $1,322.13 \pm 16.576$ & $1,307.38 \pm 16.332$ & $1,326.81 \pm 16.467$ & $1,312.16 \pm 16.385$ & \\
\hline \multicolumn{6}{|c|}{ Feed:Gain Ratio - FCR $\left(\mathrm{g} \mathrm{g}^{-1}\right)$} \\
\hline NVAC & $1.306 \mathrm{Bb}$ & $1.336 \mathrm{Bb}$ & $1.374 \mathrm{Bab}$ & $1.395 \mathrm{Ba}$ & $1.353 \pm 0.012$ \\
\hline VAC & $1.389 \mathrm{Ab}$ & $1.391 \mathrm{Ab}$ & $1.417 \mathrm{Aab}$ & $1.476 \mathrm{Aa}$ & $1.418 \pm 0.016$ \\
\hline Mean $\pm \mathrm{SE}^{*}$ & $1.347 \pm 0.012$ & $1.364 \pm 0.011$ & $1.396 \pm 0.015$ & $1.436 \pm 0.018$ & \\
\hline \multicolumn{6}{|c|}{1 to $41 \mathrm{~d}$ of age } \\
\hline \multicolumn{6}{|c|}{ Body Weight - BW (g) } \\
\hline NVAC & $2,748.83 \mathrm{Aa}$ & $2,708.58 \mathrm{Aa}$ & $2,776.49 \mathrm{Aa}$ & $2,747.44 \mathrm{Aa}$ & $2,745.33 \pm 26.45$ \\
\hline VAC & $2,690.88 \mathrm{Ba}$ & $2,680.14 \mathrm{Ba}$ & $2,691.87 \mathrm{Ba}$ & $2,660.30 \mathrm{Ba}$ & $2,680.80 \pm 25.95$ \\
\hline Mean $\pm S E^{*}$ & $2,719.86 \pm 26.624$ & $2,694.36 \pm 26.482$ & $2,734.18 \pm 26.652$ & $2,703.87 \pm 26.342$ & \\
\hline \multicolumn{6}{|c|}{ Body Weight Gain - BWG (g) } \\
\hline NVAC & $2,705.36 \mathrm{Aa}$ & $2,665.51 \mathrm{Aa}$ & $2,732.76 \mathrm{Aa}$ & $2,704.77 \mathrm{Aa}$ & $2,702.10 \pm 26.24$ \\
\hline VAC & $2,647.57 \mathrm{Ba}$ & $2,636.68 \mathrm{Ba}$ & $2,648.41 \mathrm{Ba}$ & $2,617.10 \mathrm{Ba}$ & $2,637.44 \pm 26.04$ \\
\hline Mean $\pm S E^{*}$ & $2,676.47 \pm 26.261$ & $2,651.10 \pm 26.088$ & $2,690.58 \pm 26.376$ & $2,660.94 \pm 26.234$ & \\
\hline \multicolumn{6}{|c|}{ Feed Intake - FI ( $\left.\mathrm{g} \mathrm{bird}^{-1}\right)$} \\
\hline NVAC & 4,765.11 Aab & $4,730.35 \mathrm{Ab}$ & $4,837.38 \mathrm{Aa}$ & 4,766.17 Aab & $4,774.75 \pm 55.28$ \\
\hline VAC & 4,698.46 Bab & 4,698.37 Bab & $4,686.93 \mathrm{Bb}$ & $4,703.00 \mathrm{Ba}$ & $4,696.69 \pm 54.78$ \\
\hline Mean $\pm S E^{*}$ & $4,731.79 \pm 55.151$ & $4,714.36 \pm 55.198$ & $4,762.15 \pm 55.387$ & $4,734.58 \pm 55.364$ & \\
\hline \multicolumn{6}{|c|}{ Production Efficiency Index - PEI } \\
\hline NVAC & $354.65 \mathrm{Aa}$ & $344.65 \mathrm{Ab}$ & $359.05 \mathrm{Aa}$ & $347.03 \mathrm{Aab}$ & $351.34 \pm 5.12$ \\
\hline VAC & $361.53 \mathrm{Aa}$ & $339.35 \mathrm{Aab}$ & $338.32 \mathrm{Ab}$ & $336.43 \mathrm{Ab}$ & $343.91 \pm 5.13$ \\
\hline Mean $\pm \mathrm{SE}^{*}$ & $358.09 \pm 5.189$ & $342.00 \pm 5.142$ & $348.68 \pm 5.153$ & $341.73 \pm 5.148$ & \\
\hline
\end{tabular}

a-b Different lowercase letters in the same row indicate significant differences by the test of Tukey $(p \leq 0.05)$.

${ }^{A-B}$ Different uppercase letters in the same column indicate significant differences by the test of Tukey $(p \leq 0.05)$.

*Mean \pm Standard Error; $N=150$ in each group 


\section{Sources of Trophic Action on Performance and Intestinal Morphometry of Broiler Chickens Vaccinated against Coccidiosis}

interaction $(p \leq 0.05)$ between the factors evaluated. The birds vaccinated against coccidiosis showed the worst results for all evaluated parameters, as shown by the development of the interactions presented in Table 2. Considering the effect of trophic sources, the groups fed L-GIn showed better BW and WG results compared with the controls. The groups fed the diets supplemented with Gln+Glu presented lower feed intake in relation to the groups supplemented with L-GIn and yeast. Therefore, the feed:gain ratio of the birds fed L-GIn and GIn+Glu was better than that obtained by the birds in the control groups.

Considering the entire experimental period (from 1 to $41 \mathrm{~d}$ of age), BW, BWG, FI and PEI were influenced $(p \leq 0.05)$ by the interaction between the factors evaluated. The factor vaccination directly influenced performance parameters, but at $41 \mathrm{~d}$ of age, the birds supplemented with L-Gln showed the best PEI values, followed by the groups fed diets with yeast and GIn+Glu inclusion. In the vaccinated groups, the group fed L-Gln showed an improvement of approximately $7.5 \%$ in PEl compared with the positive control group (361.53 vs. 336.43), and less than $1 \%$ for birds fed the other trophic sources compared with the control.

The morphometric characteristics of the intestinal mucosa (duodenum, jejunum and ileum) were evaluated on days 7, 14, 21 and 41. An effect of the vaccination on villus height of the segments analyzed was being observed since the starter phase: the birds vaccinated against coccidiosis presented shorter villi than the non-vaccinated birds, and this effect continued up to the end of the rearing period. The results of the morphometric analysis performed on days 21 and 41 are shown in Tables 3 and 4, respectively.

Table 3 - Effects of the interactions between factors on villus height $(\mu \mathrm{m})$, crypt depth $(\mu \mathrm{m})$ and villus: crypt of small intestine segments of 21-d-old broilers fed diets with different trophic sources and vaccinated (VAC) or not (NVAC) against coccidiosis.

\begin{tabular}{|c|c|c|c|c|c|}
\hline Characteristics & $L-G \ln (1.5 \%)$ & Gln+Glu (3.0\%) & Yeast $(0.5 \%)$ & Control & Mean $\pm \mathrm{SE}^{*}$ \\
\hline \multicolumn{6}{|l|}{ Duodenum } \\
\hline \multicolumn{6}{|l|}{ Villus height } \\
\hline NVAC & $1,507.10 \mathrm{Aa}$ & $1,410.55 \mathrm{Ab}$ & $1,358.78 \mathrm{Ab}$ & $1,411.77 \mathrm{Ab}$ & $1,422.47 \pm 7.61$ \\
\hline VAC & $1,382.63 \mathrm{Ba}$ & $1,351.16 \mathrm{Bb}$ & $1,320.46 \mathrm{Bb}$ & $1,367.46 \mathrm{Ba}$ & $1,355.24 \pm 7.07$ \\
\hline Mean $\pm S E^{*}$ & $1,448.92 \pm 11.00$ & $1,380.88 \pm 11.41$ & $1,340.41 \pm 9.51$ & $1,389.08 \pm 9.51$ & \\
\hline \multicolumn{6}{|l|}{ Crypt depth } \\
\hline NVAC & $202.60 \mathrm{Ba}$ & $197.43 \mathrm{Bb}$ & $172.62 \mathrm{BC}$ & $199.94 \mathrm{Bb}$ & $193.86 \pm 1.18$ \\
\hline VAC & $217.63 \mathrm{Ab}$ & $210.42 \mathrm{Ab}$ & $221.74 \mathrm{Aa}$ & $225.54 \mathrm{Aa}$ & $218.83 \pm 1.09$ \\
\hline Mean $\pm S E^{*}$ & $209.63 \pm 1.70$ & $205.89 \pm 1.77$ & $197.18 \pm 1.47$ & $212.54 \pm 1.47$ & \\
\hline \multicolumn{6}{|l|}{ Jejunum } \\
\hline \multicolumn{6}{|l|}{ Villus height } \\
\hline NVAC & $933.03 \mathrm{Aa}$ & $906.10 \mathrm{Ab}$ & $946.29 \mathrm{Aa}$ & $917.62 \mathrm{Ab}$ & $927.75 \pm 3.09$ \\
\hline VAC & $923.37 \mathrm{Ba}$ & $865.66 \mathrm{Bb}$ & $875.43 \mathrm{Bb}$ & $857.88 \mathrm{Bb}$ & $880.59 \pm 3.30$ \\
\hline Mean $\pm S E^{*}$ & $928.89 \pm 4.37$ & $885.88 \pm 4.67$ & $910.93 \pm 4.37$ & $887.75 \pm 4.67$ & \\
\hline \multicolumn{6}{|l|}{ Crypt depth } \\
\hline NVAC & $180.92 \mathrm{Bb}$ & $192.05 \mathrm{Ba}$ & $178.54 \mathrm{Bb}$ & $184.94 \mathrm{Bb}$ & $184.48 \pm 0.78$ \\
\hline VAC & $194.38 \mathrm{Aa}$ & $195.52 \mathrm{Aa}$ & $191.08 \mathrm{Ab}$ & $196.71 \mathrm{Aa}$ & $194.43 \pm 0.83$ \\
\hline Mean $\pm S E^{*}$ & $186.69 \pm 1.10$ & $193.78 \pm 1.18$ & $184.92 \pm 1.10$ & $190.83 \pm 1.18$ & \\
\hline \multicolumn{6}{|l|}{ Villus:Crypt ratio } \\
\hline NVAC & $5.18 \mathrm{Aa}$ & $4.73 \mathrm{Ab}$ & $5.35 \mathrm{Aa}$ & $5.00 \mathrm{Ab}$ & $5.10 \pm 0.024$ \\
\hline VAC & $4.77 \mathrm{Ba}$ & $4.46 \mathrm{Bb}$ & $4.58 \mathrm{Ba}$ & $4.38 \mathrm{Bb}$ & $4.55 \pm 0.026$ \\
\hline Mean $\pm S E^{*}$ & $5.00 \pm 0.034$ & $4.60 \pm 0.037$ & $5.03 \pm 0.034$ & $4.69 \pm 0.037$ & \\
\hline \multicolumn{6}{|l|}{ Ileum } \\
\hline \multicolumn{6}{|l|}{ Villus height } \\
\hline NVAC & $654.21 \mathrm{Ba}$ & $676.10 \mathrm{Ba}$ & $664.68 \mathrm{Ba}$ & $648.66 \mathrm{Ba}$ & $660.92 \pm 3.65$ \\
\hline VAC & $697.45 \mathrm{Aa}$ & $683.46 \mathrm{Aa}$ & $708.10 \mathrm{Aa}$ & $704.51 \mathrm{Aa}$ & $698.38 \pm 3.52$ \\
\hline Mean $\pm S E^{*}$ & $675.74 \pm 4.99$ & $680.31 \pm 4.99$ & $686.29 \pm 4.99$ & $676.59 \pm 5.33$ & \\
\hline \multicolumn{6}{|l|}{ Crypt depth } \\
\hline NVAC & $155.76 \mathrm{Bb}$ & $157.20 \mathrm{Ba}$ & $150.24 \mathrm{Bb}$ & $159.93 \mathrm{Ba}$ & $156.38 \pm 1.19$ \\
\hline VAC & $160.18 \mathrm{Ab}$ & $172.17 \mathrm{Ab}$ & $194.54 \mathrm{Aa}$ & $168.05 \mathrm{Ab}$ & $173.39 \pm 1.15$ \\
\hline Mean $\pm S E^{*}$ & $157.66 \pm 1.63$ & $165.75 \pm 1.63$ & $172.39 \pm 1.63$ & $163.99 \pm 1.75$ & \\
\hline
\end{tabular}

a-b Different lowercase letters in the same row indicate significant differences by the test of Tukey $(p \leq 0.05)$.

A-B Different uppercase letters in the same column indicate significant differences by the test of Tukey $(p \leq 0.05)$.

*Mean \pm Standard Error; $N=150$ in each group 
Table 4 - Effects of the interactions between factors on villus height $(\mu \mathrm{m})$, crypt depth $(\mu \mathrm{m})$ and villus:crypt of small intestine segments of 41 -d-old broilers fed diets with different trophic sources and vaccinated (VAC) and or not (NVAC) against coccidiosis.

\begin{tabular}{|c|c|c|c|c|c|}
\hline Characteristics & $L-G \ln (1.5 \%)$ & Gln+Glu (3.0\%) & Yeast $(0.5 \%)$ & Control & Mean $\pm \mathrm{SE}^{*}$ \\
\hline \multicolumn{6}{|l|}{ Duodenum } \\
\hline \multicolumn{6}{|l|}{ Villus height } \\
\hline NVAC & 1,547.27 Aab & 1,695.07 Aa & $1,510.57 \mathrm{Ab}$ & 1,605.62 Aab & $1,587.14 \pm 10.60$ \\
\hline VAC & 1,512.90 Bab & 1,565.66 Ba & $1,440.75 \mathrm{Bb}$ & 1,463.64 Bab & $1,496.24 \pm 11.00$ \\
\hline Mean $\pm S E^{*}$ & $1,527.63 \pm 9.60$ & $1,643.43 \pm 11.60$ & $1,464.03 \pm 10.90$ & $1,534.24 \pm 10.60$ & \\
\hline \multicolumn{6}{|l|}{ Crypt depth } \\
\hline NVAC & 199.21 Aab & $200.46 \mathrm{Aa}$ & $199.73 \mathrm{Ab}$ & $201.75 \mathrm{Aa}$ & $200.16 \pm 1.25$ \\
\hline VAC & 192.89 Bab & $199.95 \mathrm{Ba}$ & $189.06 \mathrm{Bb}$ & 191.33 Bab & $193.44 \pm 1.20$ \\
\hline Mean $\pm S E^{\star}$ & $195.60 \pm 1.20$ & $200.26 \pm 1.30$ & $192.62 \pm 1.10$ & $196.52 \pm 1.26$ & \\
\hline \multicolumn{6}{|l|}{ Jejunum } \\
\hline \multicolumn{6}{|l|}{ Villus height } \\
\hline NVAC & $1,283.05 \mathrm{Aa}$ & 1,259.72 Aa & $1,179.66 \mathrm{Ab}$ & 1,213.13 Aab & $1,233.60 \pm 11.80$ \\
\hline VAC & $1,208.99 \mathrm{Ba}$ & $1,224.82 \mathrm{Ba}$ & $1,106.96 \mathrm{Bb}$ & 1,191.99 Bab & $1,188.47 \pm 11.30$ \\
\hline Mean $\pm S E^{*}$ & $1,233.68 \pm 11.90$ & $1,242.27 \pm 11.35$ & $1,136.05 \pm 12.90$ & $1,204.77 \pm 13.01$ & \\
\hline \multicolumn{6}{|l|}{ Villus:Crypt ratio } \\
\hline NVAC & $7.26 \mathrm{Aa}$ & $7.37 \mathrm{Aa}$ & $7.61 \mathrm{Aa}$ & $7.35 \mathrm{Aa}$ & $7.40 \pm 0.07$ \\
\hline VAC & $7.16 \mathrm{Ba}$ & $6.99 \mathrm{Ba}$ & $6.87 \mathrm{Ba}$ & $7.31 \mathrm{Ba}$ & $7.09 \pm 0.06$ \\
\hline Mean $\pm S E^{*}$ & $7.19 \pm 0.07$ & $7.18 \pm 0.07$ & $7.17 \pm 0.08$ & $7.33 \pm 0.08$ & \\
\hline \multicolumn{6}{|l|}{ íleum } \\
\hline \multicolumn{6}{|l|}{ Villus height } \\
\hline NVAC & $797.60 \mathrm{Aa}$ & 693.14 Aab & $767.81 \mathrm{Aa}$ & $664.22 \mathrm{Ab}$ & $730.69 \pm 5.91$ \\
\hline VAC & $732.59 \mathrm{Aa}$ & $721.56 \mathrm{Aab}$ & $715.41 \mathrm{Ab}$ & $745.11 \mathrm{Aa}$ & $728.67 \pm 5.35$ \\
\hline Mean $\pm S E^{*}$ & $765.10 \pm 5.91$ & $705.77 \pm 5.10$ & $741.62 \pm 5.91$ & $699.06 \pm 5.40$ & \\
\hline
\end{tabular}

a-b Different lowercase letters in the same row indicate significant differences by the test of Tukey $(p \leq 0.05)$.

${ }^{A-B}$ Different uppercase letters in the same column indicate significant differences by the test of Tukey $(p \leq 0.05)$.

*Mean \pm Standard Error; $N=150$ in each group.

At $21 \mathrm{~d}$ of age, there was interaction $(p \leq 0.05)$ between the evaluated factors for all the characteristics, except for villus:crypt ratio in the duodenum and ileum (Table 3). In the duodenum and jejunum, the nonvaccinated birds presented higher villi compared with the vaccinated birds, and deep crypts were observed in the vaccinated birds relative to the non-vaccinated birds. In ileum, both villus height and crypt depth were higher in the vaccinated groups compared with those non-vaccinated. According to the trophic source factor, birds supplemented with L-Gln showed higher villi in the duodenum and jejunum compared with the groups supplemented with yeast and $G \ln +\mathrm{Glu}$, with no difference in ileal villus height.

For the morphometric characteristics, there was interaction $(p \leq 0.05)$ between the factors evaluated at $41 \mathrm{~d}$ of age for villus height in all segments, for duodenal crypt depth and jejunal villus:crypt ratio, as shown in Table 4. Non-vaccinated birds presented higher values than vaccinated birds; however, this effect was not observed for the ileal villus height. Comparing the factor trophic sources, the groups supplemented with L-Gln and Gln+Glu showed better duodenal and jejunal morphometric characteristics compared with birds supplemented with yeast.

\section{DISCUSSION}

Luquetti (2005) evaluated the effect of vaccination against coccidiosis and the supplementation of glutamine or of a probiotic in broiler diets. The author observed greater body weight gain at 21 and 28 days of age for the non-vaccinated birds relative to the vaccinated birds, suggesting an effect of vaccination per se on the integrity of the intestinal mucosa, influencing intestinal development. However, Maiorka et al. (2000) and Murakami et al. (2007) did not observe any significant differences in the performance of broilers supplemented with $1 \%$ glutamine throughout the entire rearing period.

With respect to the nucleotides, Pelícia et al. (2010) evaluated the inclusion of increasing levels of nucleotides in broiler diets, but observed no effect on performance of the birds at 7, 14, 21 and $42 \mathrm{~d}$ of age, 
corroborating the results of Zavarize et al. (2011), who did not find any improvement in the performance of broilers fed a diet supplemented with $0.04 \%$ nucleotides compared with the non-supplemented diet. On the other hand, Rutz et al. (2006) supplemented broiler diets with $0.2 \%$ nucleotides and observed a greater body weight gain and improved feed: gain ratio when compared with the group that did not receive the supplement. The authors suggested that the improved performance could be attributed to an increase in villus height, caused by the action of the nucleotides present in the yeast extract, enhancing nutrient digestion and absorption capacity due to the increase in absorptive surface area.

Kawazoe (2000) reported that the coccidioses represented mainly by $E$. acervulina and E. maxima are those that cause the greatest damage, injuring the duodenum and jejunum, with considerable destruction in the region of greater digestion and absorption of nutrients.

According to Macari \& Maiorka (2000), two phases in the life cycles of different species of Eimeria $s p$. occur in the epithelial cells of the small intestine and/ or cecum, destroying them, consequently destroying the mucosa villi. Thus, as the number of cells destroyed in the intestinal villi increases, so there is an attempt to repair the mucosa by a proliferation process (mitosis) in the crypts, which become deeper. The present study showed that this proliferation process was impaired because of the vaccination against coccidiosis at certain ages during the experimental period evaluated.

Similarly, Luquetti (2005) evaluated the effect of vaccination against coccidiosis and the addition of glutamine or of a probiotic in broiler diets. The author observed that vaccination influenced morphometric characteristics evaluated and that the inclusion of glutamine in broiler diets reduced losses of the epithelial mucosa.

In the present study, intestinal morphometry results corroborated the observations of Maiorka et al. (2000), Yi et al. (2005) and Murakami et al. (2007), who found an improvement in the morphometric characteristics of the intestinal mucosa, both in the duodenum and in the jejunum during the rearing period in broilers fed diets supplemented with glutamine, whether submitted to some form of injury or not.

The real mechanism by which glutamine could stimulate cell proliferation in the intestinal mucosa remains uncertain, but it is known that two mechanisms could be involved in this process: an increase in the exchange of sodium by hydrogen in the plasma membrane and an increase in the specific activity of the enzyme ornithine decarboxylase (Rhoads et al., 1997). Thus the effect of glutamine on the development of the intestinal mucosa suggests that this amino acid could have an important role in the maturation of the intestinal mucosa of the chicks during the first two weeks post-hatch (Maiorka et al., 2000). Glutamine and glutamate can also act as regulators of metabolic requirements, increasing protein synthesis and reducing its degradation in the skeletal muscles of growing chickens (Haussinger et al., 1994).

\section{CONCLUSIONS}

A negative effect of the vaccination against coccidiosis on broiler performance was observed, even when birds were fed diets supplemented with different trophic sources, except for production efficiency index at 41 days of age. Supplementation of diets with the trophic sources studied could be a strategy to reduce epithelial loss from the mucosa when broilers face challenges during the rearing period, considering the cost-benefit for the industry.

\section{ACKNOWLEDGEMENTS}

The authors thank São Paulo Research Foundation (FAPESP - Process number 2006/04655-8) and the National Council for Technological and Scientific Development (CNPq-Process number 17362459/2007) for their financial support.

\section{REFERENCES}

Amin HJ, Zamora SA, McMillan DD, Fick GH, Butzner JD, Parsons HG, Scott RB. Arginine supplementation prevents neocrotizing enterocolitis in the premature infant. Journal of Pediatric 2002;140(4):425-431.

Beçak W, Paulete J. Cytology and histology techniques. Rio de Janeiro: Scientific and Technical Books Editora; 1976.

Domeneghini C, Di Giancamillo A, Bosi G, Arrighi S. Can nutraceuticals affect the structure of intestinal mucosa? Qualitative and quantitative microanatomy in L-glutamine diet supplemented weaning piglets. Veterinary Reserch Commun 2006;30(3):331-342.

Haussinger D, Lang F, Gerok W. Regulation of cell function by cellular hydratation state. American Journal of Physiology 1994;267(3):E343-E355.

Jobgen WS, Fried SK, Fu WJ, Meininger CJ, Wu G. Regulatory role for the arginine-nitric oxide pathway in metabolism of energy substrates. Journal of Nutrition Biochemistry 2006;17(9):571-588.

Kawazoe U. Coccidiosis. In: Berchieri Júnior A, Macari M, editores. Avian diseases. Campinas: Facta; 2000. p.391-405.

Li $P$, Yin $Y$, Li D, Kim SW, Wu G. Amino acids and immune function. British of Journal Nutrition 2007;98(2):237-252. 
Luquetti BC. Effect of vaccination against avian coccidiosis and glutamine supplementation or prebiotics on the intestinal mucosa in broilers [thesis]. Jaboticabal (SP): Faculdade de Ciências Agrárias e Veterinárias, Universidade Estadual Paulista; 2005.

Macari M, Furlan RL, Gonzáles E. Avian physiology applied to broilers. 2a ed. Jaboticabal: Funep-Unesp; 2002

Macari M, Maiorka A. Gastrointestinal function and its impact on poultry performance. In: Conferência Apinco de Ciência e Tecnologia Avícolas; 2000; Campinas, São Paulo. Brasil. p.161-174.

Maiorka A, Fischer da Silva AV, Santin E, Borges AS, Boleli IC, Macari $M$. Influence of glutamine supplementation on performance and development of villi and crypts of the small intestine of chickens. Arquivo Brasileiro de Medicina Veterinária e Zootecnia 2000;52(5): 487-490.

Mateo $\mathrm{CD}$, Stein $\mathrm{HH}$. Nucleotides and young animal health: can we enhance intestinal tract development and immune function? Proceedings of Alltech's 20th Annual Symposium; 2004; Lexington, Kentucky. United States. p.159-170.

Mongin P. Recent advances in dietary cation-anion balance: applications. Proceeding of the Nutrition Society 1981;40(3):285-294.

Murakami AE, Sakamoto MI, Natali MRM, Souza LMG, Franco JRG. Supplementation of glutamine and vitamin E on the morphometry of the intestinal mucosa in broiler chickens. Poultry Science 2007;86(3):488495.

Pelícia VC, Sartori JR, Zavarize KC, Pezzato AC, Stradiotti AC, Araújo PC, Mituo MAO, Madeira LA. Effect of nucleotides on broiler performance and carcass yield. Brazilian Journal of Poultry Science 2010;12(1):3134

Revolledo L, Ferreira AJP. Anticoccidial. In: Palermo-Neto J, Spinosa ES, Górniak SL, editores. Applied Pharmacology for Poultry. São Paulo: Roca; 2005. p.189-199.

Rhoads JM, Argenzio RA, Chen W, Rippe RA, Westwick JK, Cox AD, Berschneider HM, Brenner DA. L-glutamine stimulates intestinal cell proliferation and activates mitogen-activated protein kinases. American Journal of Physiology 1997:272(5):G943-953.
Rossi P, Xavier EG, Rutz F. Nucleotides in animal nutrition. Revista Brasileira de Agrociência 2007; 13(1):05-12.

Rostagno HS, Albino LFT, Donzele JL, Gomes PC, Oliveira RF, Lopes DC, Ferreira AS, Barreto SLT. Brazilian tables for poultry and pigs. Composition of food and nutritional requirements. $2^{2} \mathrm{ed}$. Viçosa:UFV; 2005

Rutz F, Anciuti MA, Rech JL, Gonçalves FM, Delgado AD, Rosa ER, Zauk N, Ribeiro CLG, Silva RR, Dallmann PR. Performance and carcass traits of broilers receiving dietary yeast extract. Ciência Animal Brasileira 2006;7(4):349-355

Sakamoto, M.I. Performance, development and enzymatic activity of the intestinal mucosa of broiler chickens fed with diets supplemented with glutamine and nucleotides [thesis]. Pirassununga (SP): Faculdade de Zootecnia e Engenharia de Alimentos, Universidade de São Paulo; 2009.

SAS Institute SAS. User's Guide: Statistics. Version 9.1. $4^{\text {th }}$ ed. Cary, NC; 2002

Wang J, Cheng L, Li P, Li X, Zhou H, Wang F, Li D, Yin Y, Wu G. Gene expression is altered in piglet small intestine by weaning and dietary glutamine supplementation. Journal of Nutrition 2008; 138(6):10251032.

Wang WW, Qiao SY, Li DF. Amino acids and gut function. Amino Acids 2009;37(1):105-110.

Wu G, Bazer FW, Davis TA, Jaeger LA, Johnson GA, Kim SW, Knabe DA Meininger $C J$, Spencer TE, Yin YL. Important roles for the arginine family of amino acids in swine nutrition and production. Livestock Science 2007:112:8-22.

Yi FG, Allee GL, Knight CD, Dibner JJ. Impact of Glutamine and Oasis hatchling supplement on growth performance, small intestinal morphology, and immune response of broilers vaccinated and challenged with Eimeria maxima. Poultry Science 2005;84(2):283-293.

Zavarize KC, Sartori JR, Pelícia VC, Pezzato AC, Araújo PC, Stradiotti AC, Madeira LA. Glutamine and nucleotides in the diet of broiler chickens raised in the alternative system. Archivos de Zootecnia 2011;60(232):913-920. 